\title{
Comparative Study of some Numerical Methods to Solve a 3D Advection-Diffusion Equation
}

\author{
A. R. Appadu ${ }^{1}$, J. K. Djoko ${ }^{1}$ and H. H. Gidey ${ }^{1,2}$ \\ ${ }^{1}$ Department of Mathematics and Applied Mathematics, University of Pretoria, Pretoria, 0002, South Africa. \\ ${ }^{2}$ Department of Computational Sciences, Aksum University, Aksum, 1010, Tigray, Ethiopia.
}

\begin{abstract}
In this work, three finite difference methods have been used to solve a three dimensional advection-diffusion equation with given initial and boundary conditions. The three methods are fourth order finite difference method, Crank-Nicolson and Implicit Chapeau Function. We compare the performance of the methods by computing $L_{2}$ error, $L_{\infty}$ error and some performance indices such as mass distribution ratio (MDR), mass conservation ratio (MCR), total mass and $R^{2}$ which is a measure of total variation in particle distribution.
\end{abstract}

Keywords:three dimensional advection-diffusion equation, finite difference methods

PACS: 47.11. $-j, 02.70 .-c, 02.60 .-x$

\section{INTRODUCTION}

The advection-diffusion equation is one of the most challenging equations in science as it represents two different transport processes: advection and diffusion. It has been used to describe heat transfer in a draining film [1], mass transfer [2], flow in porous media [3] and charge transport in semi-conductor devices [4]. The 3D advection-diffusion equation is given by:

$$
\frac{\partial u}{\partial t}+\beta_{x} \frac{\partial u}{\partial x}+\beta_{y} \frac{\partial u}{\partial y}+\beta_{z} \frac{\partial u}{\partial z}=\alpha_{x} \frac{\partial^{2} u}{\partial x^{2}}+\alpha_{y} \frac{\partial^{2} u}{\partial y^{2}}+\alpha_{z} \frac{\partial^{2} u}{\partial z^{2}}
$$

where $\beta_{x}, \beta_{y}, \beta_{z}$ are the velocity components of advection in the direction of $x, y$ and $z$, respectively, and $\alpha_{x}, \alpha_{y}$, and $\alpha_{z}$ are diffusivities in the $x, y$ and $z$ directions, respectively.

Yang et al. [5] studied the accuracy of three numerical methods, namely explicit central difference, implicit CrankNicolson and implicit Chapeau function methods, for solving advection-diffusion equation as applied to spore and insect dispersal. In this work, we compare the performance of three finite difference methods, namely: fourth Order upwind scheme [6], Crank-Nicolson and Implicit Chapeau function when used to solve the partial differential equation in (1) with initial conditions

$$
u(x, y, z, 0)=\exp \left(-\frac{(x-0.5)^{2}}{\alpha_{x}}-\frac{(y-0.5)^{2}}{\alpha_{y}}-\frac{(z-0.5)^{2}}{\alpha_{z}}\right) .
$$

The analytical solution to this problem is [7]

$$
u(x, y, z, t)=\frac{1}{(4 t+1)^{3 / 2}} \exp \left[-\frac{\left(x-\beta_{x} t-0.5\right)^{2}}{\alpha_{x}(4 t+1)}-\frac{\left(y-\beta_{y} t-0.5\right)^{2}}{\alpha_{y}(4 t+1)}-\frac{\left(z-\beta_{z} t-0.5\right)^{2}}{\alpha_{z}(4 t+1)}\right] .
$$

The boundary conditions are obtained by direct substitution into Eq. (3). The same approach was done by Yang et al. [5].

To analyse and compare the performance of the three methods, we compute the $L_{2}$ and $L_{\infty}$ errors and some performance indices such as; Total mass, mass conservation ratio (MCR), mass distribution ratio (MDR) and $R^{2}$ (which is 
a measure of goodness fit) [8]. We next describe how these performances indices are calculated. Let $v_{i, j, k}$ be the exact solution and $\bar{v}$ be its mean, then $R^{2}$, Total mass, MCR and MDR are calculated as

$$
\begin{array}{r}
R^{2}=1-\frac{\sum_{i=0}^{N x} \sum_{j=0}^{N y} \sum_{k=0}^{N z}\left(v_{i, j, k}-u_{i, j, k}\right)^{2}}{\sum_{i=0}^{N x} \sum_{j=0}^{N y} \sum_{k=0}^{N z}\left(v_{i, j, k}-\bar{v}\right)^{2}}, \quad \text { Total mass }=\sum_{i=0}^{N x} \sum_{j=0}^{N y} \sum_{k=0}^{N z} u_{i, j, k}^{n}, \\
\operatorname{MCR}=\frac{\sum_{i=0}^{N x} \sum_{j=0}^{N y} \sum_{k=0}^{N z} u_{i, j, k}^{n}}{\sum_{i=0}^{N x} \sum_{j=0}^{N y} \sum_{k=0}^{N z} v_{i, j, k}^{n}}, \quad \text { MDR }=\frac{\sum_{i=0}^{N x} \sum_{j=0}^{N y} \sum_{k=0}^{N z}\left(u_{i, j, k}^{n}\right)^{2}}{\sum_{i=0}^{N x} \sum_{j=0}^{N y} \sum_{k=0}^{N z}\left(v_{i, j, k}^{n}\right)^{2}} .
\end{array}
$$

\section{NUMERICAL METHODS}

In this section, three finite difference methods are presented to solve a 3D advection diffusion equation. For simplicity, we use the following notations:

$$
c_{x}=\frac{\beta_{x} \Delta t}{\Delta x}, \quad c_{y}=\frac{\beta_{y} \Delta t}{\Delta y}, \quad c_{z}=\frac{\beta_{z} \Delta t}{\Delta z}, \quad s_{x}=\frac{\alpha_{x} \Delta t}{\Delta x^{2}}, \quad s_{y}=\frac{\alpha_{y} \Delta t}{\Delta y^{2}}, \quad s_{z}=\frac{\alpha_{z} \Delta t}{\Delta z^{2}} .
$$

\section{Implicit Crank-Nicolson Method (ICN)}

Since the 3D implicit methods are costly, we use fractional splitting methods, by splitting the 3D problem into three $1 \mathrm{D}$ equations [5].

$$
\begin{aligned}
& \frac{\partial u}{\partial t}=-\beta_{x} \frac{\partial u}{\partial x}+\alpha_{x} \frac{\partial^{2} u}{\partial x^{2}} \\
& \frac{\partial u}{\partial t}=-\beta_{y} \frac{\partial u}{\partial y}+\alpha_{y} \frac{\partial^{2} u}{\partial y^{2}} \\
& \frac{\partial u}{\partial t}=-\beta_{z} \frac{\partial u}{\partial z}+\alpha_{z} \frac{\partial^{2} u}{\partial z^{2}}
\end{aligned}
$$

At the first fractional time step, i. e., from $t_{n}$ to $t_{n+1 / 3}$, Eq. (4) is discretized by first separating into two equations as follows:

$$
\begin{aligned}
& \frac{1}{2} u_{t}=-\beta_{x} u_{x} \\
& \frac{1}{2} u_{t}=\alpha_{x} u_{x x} .
\end{aligned}
$$

These two equations are discretized as

$$
\frac{1}{2} \frac{u_{i, j, k}^{n+1 / 3}-u_{i, j, k}^{n}}{\Delta t}=-\beta_{x}\left(\theta \frac{u_{i+1, j, k}^{n+1 / 3}-u_{i-1, j, k}^{n+1 / 3}}{2 \Delta x}+(1-\theta) \frac{u_{i+1, j, k}^{n}-u_{i-1, j, k}^{n}}{2 \Delta x}\right) .
$$

and

$$
\frac{1}{2}\left(\frac{u_{i, j, k}^{n+1 / 3}-u_{i, j, k}^{n}}{\Delta t}\right)=\alpha_{x}\left(\theta \frac{u_{i+1, j, k}^{n+1 / 3}-2 u_{i, j, k}^{n+1 / 3}+u_{i-1, j, k}^{n+1 / 3}}{\Delta x^{2}}+(1-\theta) \frac{u_{i+1, j, k}^{n}-2 u_{i, j, k}^{n}+u_{i-1, j, k}^{n}}{\Delta x^{2}}\right),
$$

respectively, where $\theta \in[0,1]$ is temporal weighting factor. Combining Eqs. (9) and (10), we get

$$
A_{x} u_{i-1, j, k}^{n+1 / 3}+B_{x} u_{i, j, k}^{n+1 / 3}+C_{x} u_{i+1, j, k}^{n+1 / 3}=D_{i, j, k}^{n}
$$

where

$$
\begin{gathered}
A_{x}=-\theta c_{x}-2 \theta s_{x}, \quad B_{x}=2+4 \theta s_{x}, \quad C_{x}=\theta c_{x}-2 \theta s_{x} \\
D_{i, j, k}^{n}=(1-\theta)\left[\left(c_{x}+2 s_{x}\right) u_{i-1, j, k}^{n}+\left(-c_{x}+2 s_{x}\right) u_{i+1, j, k}^{n}\right]+\left[2-4(1-\theta) s_{x}\right] u_{i, j, k}^{n} .
\end{gathered}
$$


Following the same approach as the integration procedure in the $x$-direction, we obtain

$$
A_{y} u_{i, j-1, k}^{n+2 / 3}+B_{y} u_{i, j, k}^{n+2 / 3}+C_{y} u_{i, j+1, k}^{n+2 / 3}=D_{i, j, k}^{n+1 / 3} \text {, and } A_{z} u_{i, j, k-1}^{n+1}+B_{z} u_{i, j, k}^{n+1}+C_{z} u_{i, j, k+1}^{n+1}=D_{i, j, k}^{n+2 / 3},
$$

at the second and third fractional time steps, respectively.

\section{Implicit Chapeau function Method (ICF)}

In this section we refer to Yang et al. [5]. We follow the same procedure as that of the Crank Nicolson method where (7) and (8) are discretized, respectively by

$$
\frac{1}{12 \Delta t}\left[\left(u_{i-1, j, k}^{n+1 / 3}-u_{i-1, j, k}^{n}\right)+4\left(u_{i, j, k}^{n+1 / 3}-u_{i, j, k}^{n}\right)+\left(u_{i+1, j, k}^{n+1 / 3}-u_{i+1, j, k}^{n}\right)\right]=-\beta_{x}\left(\theta \frac{u_{i+1, j, k}^{n+1 / 3}-u_{i-1, j, k}^{n+1 / 3}}{2 \Delta x}+(1-\theta) \frac{u_{i+1, j, k}^{n}-u_{i-1, j, k}^{n}}{2 \Delta x}\right) .
$$

and

$$
\begin{aligned}
& \frac{1}{12 \Delta t}\left[\left(u_{i-1, j, k}^{n+1 / 3}-u_{i-1, j, k}^{n}\right)+4\left(u_{i, j, k}^{n+1 / 3}-u_{i, j, k}^{n}\right)+\left(u_{i+1, j, k}^{n+1 / 3}-u_{i+1, j, k}^{n}\right)\right]= \\
& \alpha_{x}\left[\theta\left(\frac{u_{i+1, j, k}^{n+1 / 3}-2 u_{i, j, k}^{n+3}+u_{i-1, j, k}^{n+1 / 3}}{\Delta x^{2}}\right)+(1-\theta)\left(\frac{u_{i+1, j, k}^{n}-2 u_{i, j, k}^{n}+u_{i-1, j, k}^{n}}{\Delta x^{2}}\right)\right] .
\end{aligned}
$$

Combining (12) and (13), we get

$$
A_{x} u_{i-1, j, k}^{n+1 / 3}+B_{x} u_{i, j, k}^{n+1 / 3}+C_{x} u_{i+1, j, k}^{n+1 / 3}=D_{i, j, k}^{n},
$$

where $A_{x}=2-6 \theta c_{x}-12 \theta s_{x}, \quad B_{x}=8+24 \theta s_{x}, \quad C_{x}=2+6 \theta c_{x}-12 \theta s_{x}$ and

$$
D_{i, j, k}^{n}=\left[2+(1-\theta)\left(6 c_{x}+12 s_{x}\right)\right] u_{i-1, j, k}^{n}+\left(8-24(1-\theta) s_{x}\right) u_{i, j, k}^{n}+\left[2+(1-\theta)\left(-6 c_{x}+12 s_{x}\right)\right] u_{i+1, j, k}^{n}
$$

Using the same procedures as in the first fractional time step for the integrations in the $y$-direction and $z$-direction, we obtain

$$
A_{y} u_{i, j-1, k}^{n+2 / 3}+B_{y} u_{i, j, k}^{n+2 / 3}+D_{y} u_{i, j+1, k}^{n+2 / 3}=D_{i, j, k}^{n+1 / 3} \text {, and } A_{z} u_{i, j, k-1}^{n+1}+B_{z} u_{i, j, k}^{n+1}+D_{z} u_{i, j, k+1}^{n+1}=D_{i, j, k}^{n+2 / 3} \text {. }
$$

\section{Fourth Order Finite Difference Method (FOM)}

Using the fourth order finite difference method [6] at the first fractional time step, we obtain

$$
u_{i, j, k}^{n+1 / 3}=A_{x} u_{i-2, j, k}^{n}+B_{x} u_{i-1, j, k}^{n}+C_{x} u_{i, j, k}^{n}+D_{x} u_{i+1, j, k}^{n}+E_{x} u_{i+2, j, k}^{n},
$$

where

$$
\begin{aligned}
& A_{x}=\frac{1}{24}\left(12 s_{x}\left(s_{x}+c_{x}^{2}\right)+2 s_{x}\left(6 c_{x}-1\right)+c_{x}\left(c_{x}-1\right)\left(c_{x}+1\right)\left(c_{x}+2\right)\right), \\
& B_{x}=-\frac{1}{6}\left(12 s_{x}\left(s_{x}+c_{x}^{2}\right)+2 s_{x}\left(3 c_{x}-4\right)+c_{x}\left(c_{x}-2\right)\left(c_{x}+1\right)\left(c_{x}+2\right)\right), \\
& C_{x}=\frac{1}{4}\left(12 s_{x}\left(s_{x}+c_{x}^{2}\right)-10 s_{x}+\left(c_{x}-1\right)\left(c_{x}-2\right)\left(c_{x}+1\right)\left(c_{x}+2\right)\right), \\
& D_{x}=-\frac{1}{6}\left(12 s_{x}\left(s_{x}+c_{x}^{2}\right)-2 s_{x}\left(3 c_{x}+4\right)+c_{x}\left(c_{x}-2\right)\left(c_{x}-1\right)\left(c_{x}+2\right)\right), \\
& E_{x}=\frac{1}{24}\left(12 s_{x}\left(s_{x}+c_{x}^{2}\right)-2 s_{x}\left(6 c_{x}+1\right)+c_{x}\left(c_{x}-1\right)\left(c_{x}+1\right)\left(c_{x}-2\right)\right) .
\end{aligned}
$$

In a similar way, we obtain

$$
u_{i, j, k}^{n+2 / 3}=A_{y} u_{i, j-2, k}^{n+1 / 3}+B_{y} u_{i, j-1, k}^{n+1 / 3}+C_{y} u_{i, j, k}^{n+1 / 3}+D_{y} u_{i, j+1, k}^{n+1 / 3}+E_{y} u_{i, j, k+2}^{n+1 / 3},
$$

and

$$
u_{i, j, k}^{n+1}=A_{z} u_{i, j, k-2}^{n+2 / 3}+B_{z} u_{i, j, k-1}^{n+2 / 3}+C_{z} u_{i, j, k}^{n+2 / 3}+D_{z} u_{i, j, k+1}^{n+2 / 3}+E_{z} u_{i, j, k+2}^{n+2 / 3} .
$$

at the second and third fractional time steps, respectively. 
TABLE 1. $h=0.05$ and $k=0.001, T=0.05$.

\begin{tabular}{ccccccccc}
\hline Method & $L_{2}$ - error & $L_{\infty}$ & Total mass & $R^{2}$ & MCR & MDR & $\min u$ & $\max u$ \\
\hline Exact & 0 & 0 & 44.5466 & 1 & 1 & 1 & $1.6640 \times 10^{-32}$ & 0.7419 \\
\hline ICN & 0.0032 & 0.0799 & 44.5465 & 0.9932 & 1 & 1.0129 & $-3.9948 \times 10^{-5}$ & 0.7145 \\
ICF & $5.8236 \times 10^{-4}$ & 0.0210 & 44.5466 & 0.9998 & 1 & 0.9870 & $-1.2857 \times 10^{-10}$ & 0.7209 \\
FOM & $8.3415 \times 10^{-4}$ & 0.0226 & 44.5466 & 0.9995 & 1 & 1.0013 & $-1.2422 \times 10^{-4}$ & 0.7330 \\
\hline
\end{tabular}

TABLE 2. $h=0.05$ and $k=0.001, T=0.2$.

\begin{tabular}{ccccccccc}
\hline Method & $L_{2}$ - error & $L_{\infty}$ & Total mass & $R^{2}$ & MCR & MDR & $\min u$ & $\max u$ \\
\hline Exact & 0 & 0 & 44.5400 & 1 & 1 & 1 & $1.2227 \times 10^{-32}$ & 0.4072 \\
ICN & 0.0052 & 0.0916 & 44.3273 & 0.9658 & 0.9952 & 1.0238 & -0.0027 & 0.4059 \\
ICF & $7.7228 \times 10^{-4}$ & 0.0163 & 44.8431 & 0.9992 & 1.0068 & 0.9778 & $-5.0893 \times 10^{-10}$ & 0.3910 \\
FOM & $9.6606 \times 10^{-4}$ & 0.0181 & 44.5894 & 0.9988 & 1.0011 & 1.0016 & $1.2227 \times 10^{-32}$ & 0.4110 \\
\hline
\end{tabular}

\section{NUMERICAL RESULTS}

The numerical results obtained using the three numerical methods at time $T=0.05$ and 0.2 are shown in Tables 1-2. For all computations, we consider the spatial step sizes $\Delta x=\Delta y=\Delta z=h=0.05$ and the temporal step size, $\Delta t=$ $k=0.001$, where the advection and diffusion coefficients are given by $\beta_{x}=\beta_{y}=\beta_{z}=0.8$ and $\alpha_{x}=\alpha_{y}=\alpha_{z}=0.01$. These three methods are stable at these values of $\Delta x, \Delta y, \Delta z, \Delta t, \beta_{x}, \beta_{y}, \beta_{z}, \alpha_{x}, \alpha_{y}$ and $\alpha_{z}$.

\section{Conclusion}

In this paper, three numerical methods have been used to solve a 3D advection-diffusion problem, with spatial step size, $h=0.05$ and temporal step size, $k=0.001$ at two different times; $T=0.05,0.2$. We compare $L_{2}, L_{\infty}, \mathrm{MCR}$, MDR, Minimum and Maximum values of $u$ using the three methods and also the corresponding exact values. We observe that as we progress in time, the maximum value of $u$ decreases as expected as the partial differential equation has dissipative terms. Table 1 shows the errors at $h=0.05, k=0.001$ and $T=0.05$. Based on $L_{2}$ and $L_{\infty}$ errors, ICF is best scheme followed by FOM followed by ICN. Based on the positive definite character, ICF is best scheme out of the three methods, though it is not completely positive definite. MDR values from ICN and FOM are respectively 1.0129 and 1.0013 while this value from ICF is 0.9870 . MDR is affected by mass spreading into areas of the grid that should remain free of mass, by damping of the peak concentration, by negative ripples or by diffusive events in the solution [9]. Table 2 shows errors at $h=0.05, k=0.001$ and $T=0.2$. Based on $L_{2}, L_{\infty}$ errors, MDR values, most efficient scheme is ICF followed by FOM. We conclude that in general ICF is quite an efficient method to solve the problem.

\section{Acknowledgments}

Dr Appadu is grateful to the South African DST/NRF SARChI Chair on Mathematical Models and Methods in Bioengineering and Biosciences of the University of Pretoria and to the National Research Foundation of South African Grant Number 95864. Prof. J.K. Djoko is funded through the incentive fund N00 401 Project 85796. Hagos is grateful to DST-NRF Centre of Excellence in Mathematical and Statistical Sciences (CoE-MaSS) for the financial support for 2016.

\section{REFERENCES}

[1] J. Isenberg and Gutfinger, International Journal of Heat Transfer 16, 505-513 (1972).

[2] V. Guvanasen and R. Volker, International Journal for Numerical methods in Fluids 3, 103-123 (1983).

[3] N. Kumar, Journal of Hydrology 63, 343-358 (1983).

[4] M. Ehrhardt and R. E. Mickens, Applied Mathematics and Computations 219, 6591-6604 (2013).

[5] Y. Yang, L. T. Wilson, M. E. Makela, and M. A. Marchetti, Ecological Modeling 109, 1-24 (1998).

[6] M. Dehghan, Mathematical Problems in Engineering 1, 61-74 (2005).

[7] M. Dehghan, Applied Mathematics and Computation 150, 5-19 (2004).

[8] T. O. Kvalseth, The American Statistician 39, 279-285 (1985).

[9] K. Schere, Atmospheric Environment 17, 1897-1907 (1983). 